\title{
GLOBAL PHOTOMETRIC CAMPAIGNS ON PULSATING
}

\section{PLANETARY NUCLEI}

\author{
ROBIN CIARDULLO \\ Pennsylvania State University \\ University Park, Pennsylvania USA \\ and \\ HOWARD E. BOND \\ Space Telescope Science Institute \\ Baltimore, Maryland USA
}

As reported in a contributed paper at this symposium, six "O VI"-type planetary nuclei are known to be pulsating variables. Through the techniques of asteroseismology, it should be possible to explore the interior structures of these stars, determine their masses and rotation rates, and measure the stellar evolutionary timescales through observations of changes in the pulsation periods.

In 1991 November and 1992 May, we and worldwide networks of collaborators carried out CCD photometry of the pulsating nuclei of NGC 1501 and Sanduleak 3, respectively, for continuous 10 -day intervals.

The data analysis for both campaigns is still underway, so this paper represents a progress report.

Collaborators for the NGC 1501 campaign (1991 November 14-27) were as follows: Kitt Peak National Observatory 0.9-m: H. E. Bond; Lick Observatory 1.0-m: R. Stover; Nishiharima Observatory 0.6-m: T. Kuroda, T. Ishida, et al.; Okayama Observatory 0.9-m: H. Malasan, S. Tamura, A. Yamasaki, E. Kambe, et al.; Ouda Observatory 0.6-m: T. Kato, R. Hirata, et al.; Beijing Observatory 2.2-m: J.-S. Chen; Wise Observatory 1.0-m: E. Leibowitz; Wendelstein Observatory 0.8-m: M. Roth, T. Soffner; Data Reduction: R. Ciardullo; Theory: S. Kawaler (Iowa State University).

Due to the favorably high declination of NGC 1501, the coverage was good: 218 hours over the interval 1991 Nov $14.5-27.5$ (70\% coverage).

The light curve of the central star was very irregular, with pulsation amplitudes of up to $\sim 0.1 \mathrm{mag}$ (peak-to-peak), but usually smaller. The power spectra are dominated by two peaks at 0.758 and $0.866 \mathrm{mHz}$ (periods of 1318 and $1154 \mathrm{sec}$ ), but there are many lower-amplitude modes as well. The amplitude of the 1154-sec mode appears to vary with a period of $\sim 8-9$ days, suggesting it is composed of at least two closely spaced components. Data reduction and analysis for the complete set of observations are underway.

Collaborators for the Sanduleak 3 campaign (1992 May 4-15) were as follows: Cerro Tololo Interamerican Observatory 0.9-m: H. E. Bond; Mt. John Observatory 1.0-m: D. Sullivan, W. Tobin, M. Clark, J. Pritchard; Kavalur Observatory 1.0-m: M. Parthasarathy; South African Astr. Obs. 1.0-m: D. Buckley; Data Reduction: R. Ciardullo; Theory: S. Kawaler.

During 1992 May 4.5-15.5, we obtained 155.5 hours of data, or $59 \%$ coverage. Detailed analysis of the data was just beginning at this writing. 